\title{
Unanticipated Intraoperative Endotracheal Tube Switch during Skull Base Surgery
}

\author{
Dheeraj Masapu $^{1} \quad$ H. R. Sunil Kumar ${ }^{1} \quad$ Munireddy Manjunath ${ }^{1}$ Satish Rudrappa ${ }^{1}$
}

\author{
${ }^{1}$ Department of Neurosciences and Sakra World Hospital, Bengaluru, \\ Karnataka, India
}

J Neuroanaesthesiol Crit Care 2018;5:34-36.

During complex maxillofacial surgeries, there may arise a need for change of endotracheal tube (ETT) route from transnasal position to transoral position to improve surgical visibility. It is usually a planned procedure, under controlled conditions with clear airway. It can be accomplished by removing the nasotracheal tube and replacing it with an orotracheal tube using intubat ing devices after completion of transoral resection. This could be challenging if the skull base is exposed, oral cavity is filled with blood and with the presence of an open maxilla in a patient who is on a rigid pinion fixation. There is a high risk of losing the airway, aspiration of oral contents, central nervous system infection, etc.
Address for correspondence Dheeraj Masapu, MD, DM, Department of Neurosciences, Sakra World Hospital, Beside Intel Office, Bellandur, Bengaluru 560 034, Karnataka, India (e-mail: dheerajmasapu@gmail.com).

A 30-year-old male patient presented with a history of double vision and headache for 3 months with otherwise normal neurological examination. His magnetic resonance imaging revealed a large well-defined lobulated extra-axial mass lesion in the sphenoid clival region measuring $6.7 \mathrm{~cm} \times 4.8 \mathrm{~cm} \times 3.0 \mathrm{~cm}$, extending into the sphenoid sinus. Midfacial approach with Le fort 2 incision was planned - Fig. 1. Nasal intubation was requested by the maxillofacial surgeon; hence, the - Fig. 2 patient was intubated nasally with a $7.5 \mathrm{~mm}$ internal diameter (ID) cuffed flexometallic ETT. After the resection of maxilla, neurosurgeon opined that the approach through the midfacial plane alone woul d yield suboptimal resection of a tumor, while transnasal transsphenoidal

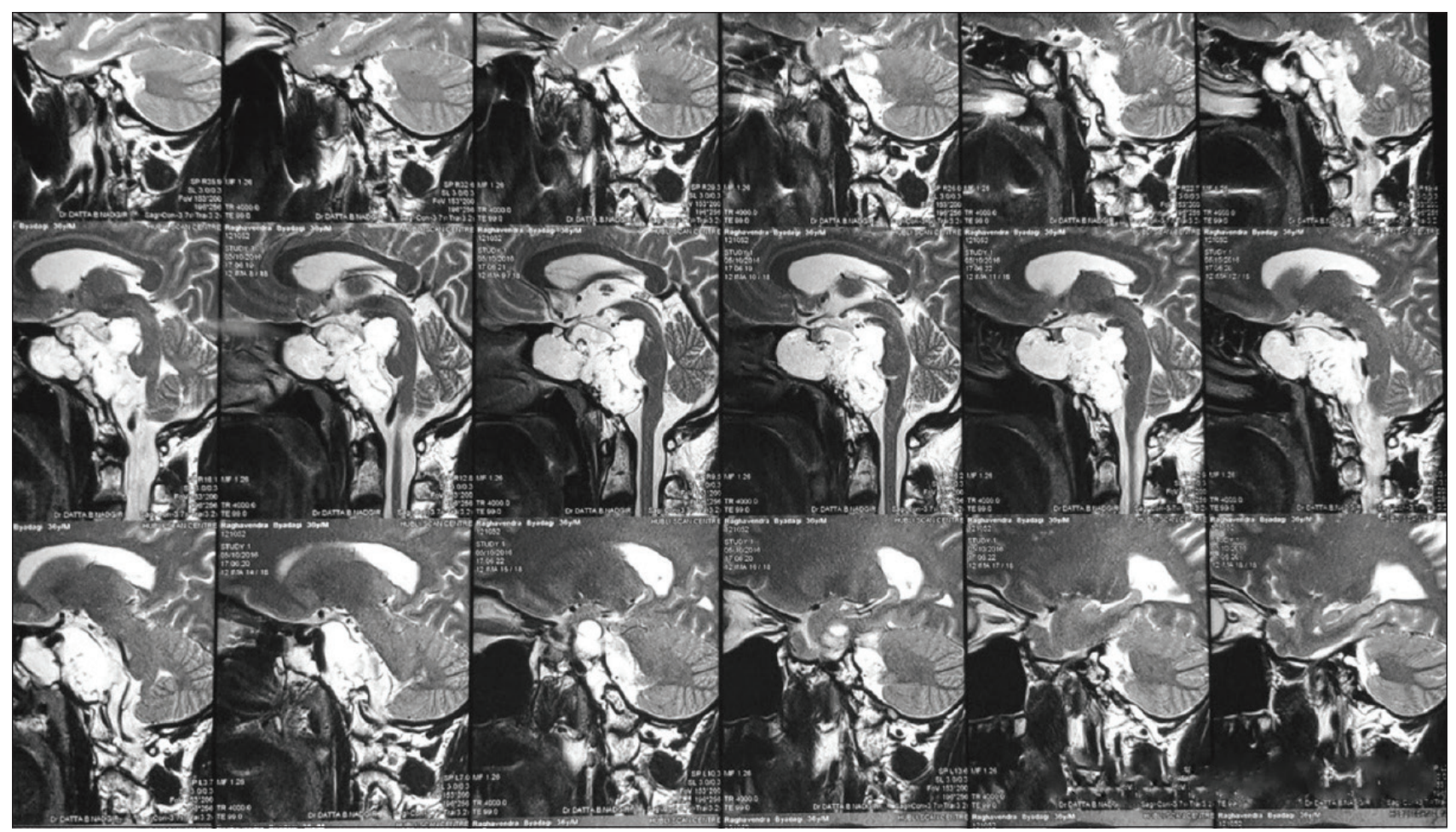

Fig. 1 Magnetic resonance imaging showing the lesion.

DOI https://doi.org/

$10.1055 / \mathrm{s}-0037-1618324$ ISSN 2348-0548.
Copyright $\odot 2018$ Indian Society of Neuroanaesthesiology and Critical Care
License terms

(9)(1) $\Theta \circledast$ 


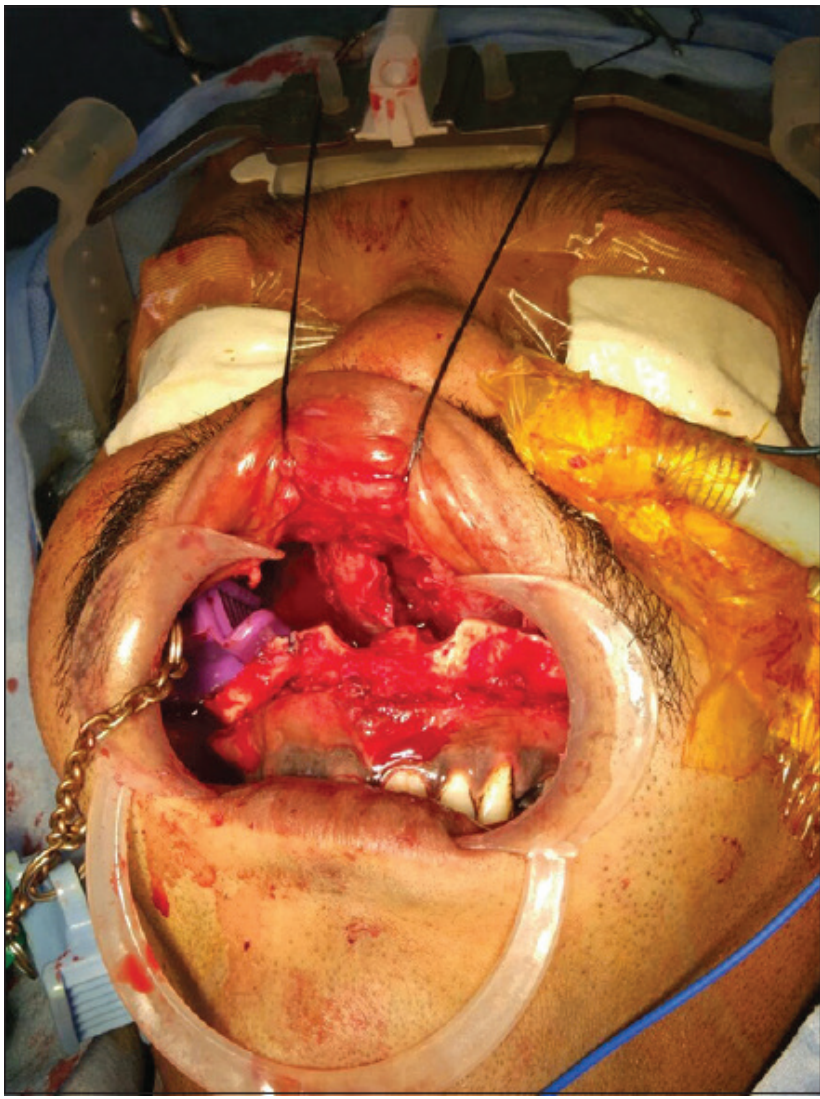

Fig. 2 Before exchange of the tube with Le fort 2 exposure.

approach will result in better resection. Hence, he requested a change of ETT from nasal to oral route so as to facilitate optimal exposure. This was challenging as there were multiple risks including loss of airway, aspiration, infection, etc. It was also technically challenging to maintain sterility due to the presence of throat pack and loose maxilla in the oral cavity.

Under strict aseptic precautions, throat pack was removed after thorough suctioning. An intubating bronchoscope was passed into the glottic area so as to confirm adequate visualisation in rigid pinion fixation.

After confirming the accessibility and adequate pre-oxygenation, a ventilating bougie was inserted through the nasal ETT into trachea. Then, a bronchoscope with an overriding 8.0 ID cuffed flexometallic ETT was inserted orally up to the glottic opening under bronchoscopic vision. The nasal ETT was then withdrawn over the bougie till oropharynx. The bronchoscope was then advanced into the trachea and ETT (oral) was advanced over the scope. The position of the oral ETT was confirmed visually, and the nasal tube and bougie were removed. Oral ETT was placed retromolar and secured to the cheek with a suture, and a throat pack was placed - Fig. $\mathbf{3}$.

Very few cases of tube switch during surgery were reported. Werther et $\mathrm{al}^{1}$ have reported 10 cases, wherein they switched ETTs by pushing the nasal portion of the ETT posteriorly into the nasopharynx and pulling it through the oral cavity.

Muto et $\mathrm{al}^{2}$ improved the technique using a specially designed retractor and clamp. The disadvantage of these techniques is that the change of tube to another type like

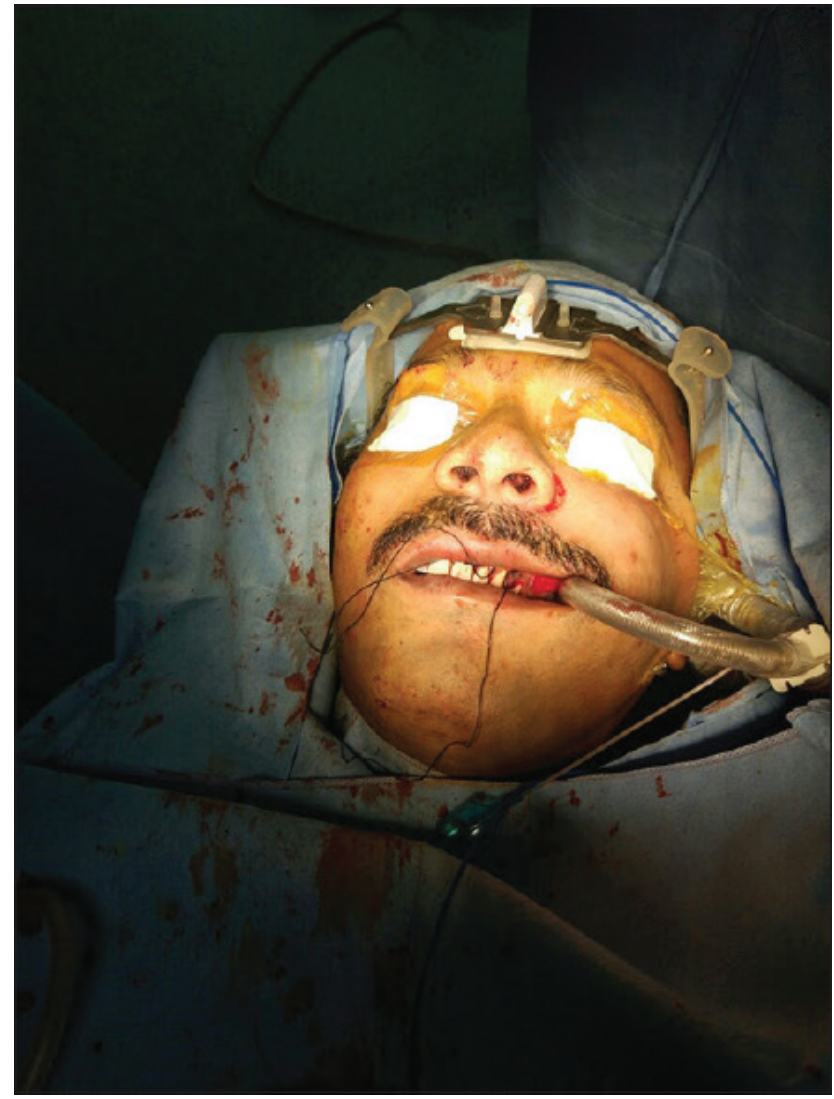

Fig. 3 After exchange of the tube.

flexometallic cannot be done and also can have difficulties with the pulling of pilot balloon assembly.

Lee et $\mathrm{al}^{3}$ used airway exchange catheter (AEC) to be pulled out of the oral cavity. The advantage with this technique is that the type of tube can be changed and also pulling of AEC is easier than pulling the tube.

The other possible options are planned submental intubation, retrograde intubation, using C-Mac or other video laryngoscope for oral intubation and tracheostomy. Many of them are invasive, while others may not guarantee definitive intubation and secure airway without disturbing the sterility of the surgical field.

We would like to conclude that it is possible to switch a nasal ETT to an oral one safely using the intubating bronchoscope, with guaranteed success in a less invasive way.

\section{Declaration of Patient Consent}

The authors certify that they have obtained all appropriate patient consent forms. In the form the patient(s) has/have given his/her/their consent for his/her/their images and other clinical information to be reported in the journal. The patients understand that their names and initials will not be published and due efforts will be made to conceal their identity, but anonymity cannot be guaranteed.

\section{Funding}

None. 


\section{Conflict of interest}

None.

\section{References}

1 Werther JR, Richardson G, Mcllwain MR. Nasal tube switch: Converting from a nasal to an oral endotracheal tube without extubation. J Oral Maxillofac Surg 1994;52:994-996
2 Muto T, Akizuki K, Wolford LM. Simplified technique to change the endotracheal tube from nasal to oral to facilitate orthognathic and nasal surgery. J Oral Maxillofac Surg 2006;64:1310-1312

3 Lee SH, Kim JE, Kang JM. Alternative technique for changing from nasal to oral endotracheal tube for orthognathic and nasal surgery by using an airway exchange catheter: a case report. Korean J Anesthesiol 2014;67:48-51 